\title{
Weiterbildung als Attraktivitätsfaktor zur Wahl eines medizinischen Fachgebiets - Anspruch und Realität
}

\author{
Die Weiterbildung ist mitentscheidend für die Wahl des medizinischen Fachgebiets \\ und den Verbleib in demselben. Konfrontiert mit dem Mangel an nachfolgenden \\ Kolleginnen und -kollegen tut die entsprechende Fachgesellschaft gut daran, hier \\ einen Hebel für die Steigerung der Attraktivität des Fachs anzusetzen. Dabei müssen \\ die mit einer modernen Erwachsenenbildung verbundenen Ansprüche nicht immer \\ der individuell erlebten Realität entsprechen, wie eine kürzlich durchgeführte \\ Befragung von Assistenzärzten im Fachgebiet Psychiatrie und Psychotherapie \\ gezeigt hat.
}

Taskforce Nachwuchsmangel $S G P P / S V P C^{*}$

\footnotetext{
* Daniel Bielinski (Leiter), Katharina Eisenhardt, Beat Frauenfelder, Denise Fels, Christoph Gitz, Jan Gysi, Daniela Krneta, Viktor Meyer, Erich Seifritz, Werner Strik
}

Die Taskforce Nachwuchsmangel der Schweizerischen Gesellschaft für Psychiatrie und Psychotherapie (SGPP) und der Schweizerischen Vereinigung Psychiatrischer Chefärztinnen und Chefärzte (SVPC) hat sich zum Ziel gesetzt, die Nachwuchssituation auf den verschiedenen Karrierestufen von der Auswahl des Medizinstudiums bis zur Tätigkeit als niedergelassener Psychiater oder Spitalpsychiaterin zu analysieren und geeignete Verbesserungsmassnahmen für die Vorstände auszuarbeiten [1].

Im Nachgang zu einer im letzten Jahr durchgeführten Befragung der psychiatrischen Kliniken und Dienste zur Nachwuchssituation nahmen im Sommer dieses Jahres in einer neuerlichen schriftlichen Befra- sönlichen Lebenserfahrungen. Als wesentliche Motivatoren für den Verbleib im Fachgebiet werden die spezifische Arbeit mit den Patienten, die bio-psychosozialen Therapiemöglichkeiten sowie die Arbeitsbedingungen am häufigsten genannt.

Die Weiterbildung steht als Motivationsfaktor hinten an. Für vier von fünf Antwortenden ist die Weiterbildung zu aufwendig bzw. komplex, zu lang oder wird den Anforderungen an eine Erwachsenenbildung nicht gerecht. Ein gleich hoher Anteil der Antwortenden vertritt zudem die Meinung, dass die Ausgestaltung der Weiterbildung Interessierte vom Fachgebiet Psychiatrie und Psychotherapie abhalte. Besonders schwer wiegt die Aussage, dass in rund
Korrespondenz:

Dr. med. Daniel Bielinski, M.H.A.

Vizepräsident SGPP

Postgasse 17

Postfach 686

CH-3000 Bern 8

\section{Die Ausgestaltung der Weiterbildung hält Interessierte vom Fachgebiet Psychiatrie und Psychotherapie ab.}

gung rund 200 Assistenzärztinnen und -ärzte aus dem Blickwinkel direkt Beteiligter Stellung zu dieser Thematik.

Jede bzw. jeder zweite Befragte entscheidet sich während der Assistenzzeit für eine Weiterbildung in Psychiatrie und Psychotherapie und nicht vor oder während des Studiums. Ausschlaggebende Faktoren für die Wahl sind dabei neben dem primären Interesse an der Psyche die Arbeitsbedingungen (Teilzeit, Kontakt mit Menschen, interdisziplinäre Zusammenarbeit), die Erfahrungen im Studium sowie die per- einem von drei Fällen die Weiterbildung der Hauptgrund für den erfolgten Absprung war. In den Erläuterungen/Bemerkungen ist etwa die Rede von «Verschulung», von inadäquater, wenig transparenter Weiterbildung, von der grossen zeitlichen Belastung neben der eigentlichen Arbeitszeit, von langen Verschiebungswegen für kurze Weiterbildungssequenzen mit zweifelhaftem didaktischem Gehalt, von Unschärfe bzw. fehlender Übersichtlichkeit und schliesslich von im Vergleich mit anderen Fachbereichen unverhältnismässig hohen Kosten für die Kandidaten, 
Vor allem die Kostenbelastung durch Selbsterfahrung und Supervision steht bei der Psychotherapie-Weiterbildung in der Kritik.

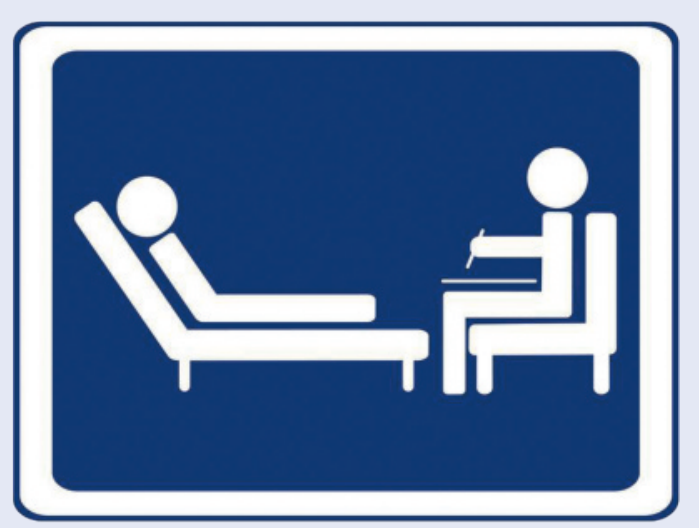

gepaart mit der unzureichenden Kostenbeteiligung durch den Arbeitgeber. Das Kostenargument wiegt je nach familiärer Situation besonders schwer.

Ungeachtet der Tatsache, dass die vorliegenden Befragungsergebnisse streng wissenschaftlich nicht repräsentativ sind und dass sich Unzufriedenheit tendenziell stärker artikuliert als Zufriedenheit, verdienen diese Analyseresultate Aufmerksamkeit. Dies besonders auch deshalb, weil sich diese Resultate mit Erfahrungen von Exponenten der Gesellschaften im direkten Kontakt mit Kolleginnen und Kollegen in der Weiterbildung decken.

Im Bestreben, die Attraktivität des Fachs zu heben, muss dementsprechend ein besonderes Augenmerk auf die Weiterbildung gelegt werden. Die drei häufigsten Vorschläge von Seiten der direkt Beteiligten:

- Die Kostenbelastung der Weiterbildungskandidaten für die Psychotherapie-Weiterbildung ist zu reduzieren. Im Fokus stehen dabei insbesondere die Selbsterfahrung und die Supervision. Zudem wird moniert, dass eine verstärkte Kostenbeteiligung durch den Arbeitgeber die Verantwortung der Weiterbildungsstätte für die Qualität der ausgewählten Psychotherapie-Weiterbildung unterstreichen würde; auch weil dadurch das Schaffen entsprechender, praxisnaher Rahmenbedingungen gefördert würde.

- Das Curriculum soll durch eine noch verstärkte Ausrichtung an der Praxis (z.B. «Bedside teaching») neu geordnet, vereinfacht und in Kombination mit neuen, elektronischen Lernformen besser am Arbeitsplatz integriert werden. So sollen etwa weite Teile der Psychotherapie-Weiterbildung in die Weiterbildungsstätten integriert werden.

- Die Weiterbildung ist zeitlich zu straffen und weitgehend während der Arbeitszeit zu absolvieren (Weiterbildung «on the job»).

Gut ein Jahr nach der Inkraftsetzung des revidierten Weiterbildungsprogramms sehen sich die Fachgesellschaft SGPP und die Chefärztevereinigung SVPC in Zusammenarbeit mit der noch relativ jungen Vereinigung Psychiatrischer Assistenzärzte und -ärztinnen SVPA auf verschiedenen Ebenen der Nachwuchsproblematik herausgefordert, aber auch in ihren umgehend initiierten Projekten bestätigt. Ausgehend von einer Analyse des Bedarfs an berufstätigen Psychiaterinnen und Psychiatern in Funktion der gestützt auf Bevölkerungsentwicklungsszenarien zu behandelnden Personen, über die geplanten Massnahmen zur Nachwuchsförderung rund um das Studium bis hin zur Prüfung neuer Modelle zur Ausgestaltung und Finanzierung der Weiterbildung, spannt sich ein weiter Bogen. Bleibt zu hoffen, dass die verschiedenen Massnahmen rechtzeitig und hinreichend greifen werden.

\section{Literatur}

1 Taskforce Nachwuchsmangel SGPP/SVPC. Schweizer Nachwuchs fehlt speziell in der Psychiatrie. Schweiz Ärztezeitung. 2010;91(10):389-90. 